\title{
Inter-Population Variability of Endosymbiont Densities in the Asian Citrus Psyllid (Diaphorina citri Kuwayama)
}

\author{
Chia-Ching Chu ${ }^{1}$ - Torrence A. Gill ${ }^{1} \cdot$ Mark Hoffmann $^{3} \cdot$ Kirsten S. Pelz-Stelinski $^{1,2}$
}

Received: 29 October 2015 / Accepted: 22 January 2016/Published online: 4 February 2016

(C) The Author(s) 2016. This article is published with open access at Springerlink.com

\begin{abstract}
The Asian citrus psyllid (Diaphorina citri Kuwayama) is an insect pest capable of transmitting Candidatus Liberibacter asiaticus (CLas), the causal agent of citrus greening in North America. D. citri also harbors three endosymbionts, Wolbachia, Candidatus Carsonella ruddii, and Candidatus Profftella armatura, which may influence D. citri physiology and fitness. Although genomic researches on these bacteria have been conducted, much remains unclear regarding their ecology and inter-population variability in $D$. citri. The present work examined the densities of each endosymbiont in adult $D$. citri sampled from different populations using quantitative PCR. Under field conditions, the densities of all three endosymbionts positively correlated with each other, and they are associated with $D$. citri gender and locality. In addition, the infection density of $C$ Las also varied across populations. Although an analysis pooling $D$. citri from different populations showed that $C$ Las-infected individuals tended to have lower endosymbiont densities compared to uninfected individuals, the difference was not significant when the population was included as a factor in the analysis, suggesting that other population-specific factors may have stronger effects on
\end{abstract}

Electronic supplementary material The online version of this article (doi:10.1007/s00248-016-0733-9) contains supplementary material, which is available to authorized users.

Kirsten S. Pelz-Stelinski

pelzstelinski@ufl.edu

1 Citrus Research and Education Center, University of Florida, Lake Alfred, FL, USA

2 Department of Entomology and Nematology, University of Florida, Gainesville, FL, USA

3 Department of Plant Sciences, University of California, Davis, Salinas, CA, USA endosymbiont densities. To determine whether there is a genetic basis to the density differences, endosymbiont densities between aged $C$ Las-negative females of two $D$. citri populations reared under standardized laboratory conditions were compared. Results suggested that inter-population variability in Wolbachia infection density is associated with the genotypes of the endosymbiont or the host. Findings from this work could facilitate understanding of $D$. citri-bacterial associations that may benefit the development of approaches for managing citrus greening, such as prevention of CLas transmission.

Keywords Huanglongbing · Intracellular endosymbionts · Primary endosymbionts $\cdot$ Bacteriome

\section{Introduction}

Bacterial endosymbionts of insects can profoundly influence their host's biology [1-3]. Some bacteria provide nutritional or protective benefits to the host, while others can affect an insect vector's efficiency in transmitting viruses [4-8]. Such findings suggest that insect-microbe associations may be exploited for managing insect pests or insect-transmitted diseases.

Citrus greening is a destructive plant disease caused by the Alphaproteobacterium Candidatus Liberibacter asiaticus (CLas) and other closely related species [9-12]. The Asian citrus psyllid (Diaphorina citri Kuwayama) transmits CLas in North America, Asia, and Brazil [10]. The sap-sucking $D$. citri can acquire CLas while feeding on infected citrus. After entering the vector insect, the bacteria can colonize and propagate in its digestive tract. Thereafter, the pathogen may cross the gut barrier and invade the salivary gland, thereby enabling $D$. citri to transmit the pathogen to another plant 
$[13,14]$. In addition to this host-microbe association, $D$. citri also harbors three endosymbionts: (1) a species of Wolbachia, a group of Alphaproteobacteria commonly found in arthropod species $[2,7,8,15,16],(2)$ the Gammaproteobacterium Candidatas Carsonella ruddii, an endosymbiont which may provide nutritional benefits to its host $[17,18]$ and (3) Candidatus Profftella armatura, a Betaproteobacterium capable of producing a defensive polyketide (diaphorin) [19]. In addition to their potential influence on $D$. citri biology, Profftella and Wolbachia's relative abundances in D. citri correlate with abundance of $C$ Las [20], suggesting that uncharacterized, direct or indirect, interactions may exist among $C$ Las and these endosymbionts.

Infection density contributes to an endosymbiont's influence on its host [21]. Previous studies on D. citri endosymbionts have either measured the endosymbionts' relative abundance (rather than absolute densities), compared endosymbiont abundance without normalization against $D$. citri DNA, focused on single populations or only targeted one endosymbiont species $[16,20,22]$. Thus, much of the associations among endosymbionts, their host, and other ecological factors remain unclear. Characterizing host-microbe associations in field $D$. citri is important not only for understanding its general biology but also for application purposes; the possibility of exploring host-bacterial interactions for $C$ Las control (i.e., paratransgenic approaches) has been proposed and studied $[23,24]$. Interestingly, a previous study showed that Wolbachia density could vary among field populations of D. citri [16]. Although we could not rule out the possibility that $D$. citri age and gender contribute to such variation [22], it is unknown whether other population-specific factors (i.e., CLas infection, genetic variation) are also associated with these patterns and whether other $D$. citri endosymbionts may exhibit similar density variability across populations.

The present study investigated whether insect-microbe associations vary across $D$. citri populations by comparing the densities of Wolbachia, Carsonella, and Profftella in D. citri adults sampled from three locations in Florida. This research also hypothesized that either: (1) high endosymbiont densities exclude CLas in D. citri, or (2) endosymbiont presence facilitates colonization of $D$. citri by $C$ Las. To evaluate these hypotheses, the effects of gender, population (locality), and infection by $C$ Las on endosymbionts densities was examined. Alternatively, this study also investigated (3) whether genetic differences among $D$. citri or endosymbiont populations contribute to the inter-population variability of endosymbiont densities. These variations were evaluated using aged D. citri cultures from geographically separated populations reared under standardized conditions. Results from this study indicated that the densities of all three endosymbionts are population dependent, and that there is a genetic basis to the population effect on Wolbachia density. CLas infection in D. citri was also found more associated with the population differences than with the endosymbiont densities. Findings from this study could improve general and applied understanding of $D$. citri biology to manage the worldwide and devastating citrus greening disease.

\section{Methods}

\section{Insect Materials}

To investigate endosymbiont densities in field $D$. citri, adult insects $(n=123)$ were sampled from three locations in Florida: LaBelle $\left(26^{\circ} 41^{\prime} 37.0^{\prime \prime} \mathrm{N}, 81^{\circ} 27^{\prime} 22.3^{\prime \prime} \mathrm{W}\right)$, Lake Alfred $\left(28^{\circ} 05^{\prime}\right.$ $\left.42.0^{\prime \prime} \mathrm{N}, 81^{\circ} 43^{\prime} 19.2^{\prime \prime} \mathrm{W}\right)$, and Clermont $\left(28^{\circ} 39^{\prime} 41.8^{\prime \prime} \mathrm{N}, 81^{\circ} 44^{\prime}\right.$ $48.4^{\prime \prime} \mathrm{W}$ ) during June 2015 (Table 1). To prevent possible influence of extreme temperatures on $D$. citri endosymbiont growth, we avoided exposing the insects to sunlight or keeping them on ice during transportation to the laboratory. After determining each $D$. citri gender, individuals were separately placed in microcentrifuge vials, flash frozen with liquid nitrogen, and stored at $-80{ }^{\circ} \mathrm{C}$.

Comparisons of endosymbiont densities were made between two laboratory cultures collected from Lake Alfred and Clermont Florida (Table 1) in order to control for variation associated with age, diet, and environment. The two cultures were reared on Murraya koenigii for over 2 months (approximately three generations) to reduce the proportion of $C$ Lasinfected psyllids. $M$. koenigii is a host plant of $D$. citri, but not $C$ Las [25]. Adult $D$. citri from these colonies were allowed to oviposit on 'Swingle' citrumelo [Citrus paradisi MacFaden $\times$ Poncirus trifoliata (L.) Raf.] placed in separate
Table 1 Details of Florida Diaphorina citri populations used in this study

\begin{tabular}{lllll}
\hline Population & Sampling site & Total D. citri sampled & Females & Males \\
\hline LaBelle (field) & $26^{\circ} 41^{\prime} 37.0^{\prime \prime} \mathrm{N}, 81^{\circ} 27^{\prime} 22.3^{\prime \prime} \mathrm{W}$ & 42 & 18 & 24 \\
Lake Alfred (field) & $28^{\circ} 05^{\prime} 42.0^{\prime \prime} \mathrm{N}, 81^{\circ} 43^{\prime} 19.2^{\prime \prime} \mathrm{W}$ & 41 & 14 & 27 \\
Clermont (field) & $28^{\circ} 39^{\prime} 41.8^{\prime \prime} \mathrm{N}, 81^{\circ} 44^{\prime} 48.4^{\prime \prime} \mathrm{W}$ & 40 & 17 & 23 \\
Lake Alfred (colony) & Laboratory & 12 & 12 & 0 \\
Clermont (colony) & Laboratory & 10 & 10 & 0 \\
\hline
\end{tabular}


screen cages $(24 \times 12 \times 12 \mathrm{in}$. $)$ in a greenhouse at $28 \pm 1^{\circ} \mathrm{C}, 50$ $\pm 10 \% \mathrm{RH}$ to reflect typical field conditions. The original (parent) D. citri adults were removed following egg hatch. One-day-old adult female offspring were sampled daily over for 6 days. Females were sampled because they tend to exhibit greater inter-population difference in endosymbiont densities compared to males, as described below. Each individual was flash frozen and stored at $-80{ }^{\circ} \mathrm{C}$ as described above. Laboratory-reared insects that were not infected with $C$ Las (Lake Alfred, $n=12$; Clermont, $n=10$; Table 1) were used in the experiments described below.

DNA from each sample was extracted following a brief wash with $70 \%$ alcohol using the DNeasy Blood \& Tissue Kit (QIAGEN, Inc., Valencia, CA). DNA samples were quantified using a NanoDrop 2000 spectrophotometer (Thermo Fisher Scientific, Lafayette, CO) and diluted to $15 \mathrm{ng} \mathrm{\mu l}^{-1}$ for subsequent quantitative real-time polymerase chain reaction (qPCR) analysis.

\section{Construction of qPCR Standard Curves}

Template DNA was prepared for conserved genes of $D$. citri and each of its endosymbionts, including the $D$. citri wingless gene, the 16S rDNA of CLas, Profftella, and Carsonella, and the Wolbachia fts $Z$ gene (Table 2). To obtain DNA templates required for $\mathrm{qPCR}$ standard curves, plasmids containing the targeted gene fragments were constructed. $16 \mathrm{~S}$ rDNA and $f t s Z$ gene fragments were amplified using primers and PCR conditions described previously (Table 2) [22, 26-28]. Gene fragments were cloned into the pGEM-T easy vector (Promega, Inc., Madison, WI, USA) and transformed into E. coli strain
JM109 following the manufacturer's instructions. Plasmids for the CLas $16 \mathrm{~S}$ rDNA and $D$. citri wingless gene were prepared as described in Coy et al. [26] using pGEM-T easy vectors. Plasmids were purified from overnight cultures of transformed JM109 strains using the QIAprep Spin Miniprep Kit (QIAGEN, Inc., Valencia, CA). Afterwards, $1 \mu \mathrm{g}$ of each plasmid was digested using PstI (New England Biolabs, Inc., Beverly, MA) following the manufacturer's instructions (in $50 \mu \mathrm{l}$ reactions). Linearized plasmids were purified from the digestion reactions using the QIAquick PCR Purification Kit (QIAGEN, Inc., Valencia, CA) and underwent serial dilutions for qPCR standard curve assays.

\section{Quantitative PCR Assays}

Both the Taqman probe (CLas and wingless, multiplex qPCR) and SYBR green methods (Carsonella, Profftella, Wolbachia) were used in this work (Table 2). To assure that quantifications of the same target genes were comparable across different qPCR plates, all samples were analyzed using standard curves constructed from the same preparation of linearized plasmid template. Quantitative PCR assays were conducted using an Applied Biosystems 7500 Fast Real-Time PCR System and the SYBR Green PCR Master Mix (Applied Biosystems, Inc., Foster City, CA) or the PerfeCTa qPCR ToughMix, Low ROX (Quanta BioSciences, Inc., Gaithersburg, MD; for Taqman assays). Final primer and probe concentrations were $0.2 \mu \mathrm{M}$ (for Carsonella and Profftella measurements) or $0.1 \mu \mathrm{M}$ (for wingless, CLas and Wolbachia assays). One microliter (15 ng) of $D$. citri template DNA was used in a $25 \mu \mathrm{l}$ qCR reaction volume. Three technical replicates were performed for each

Table 2 Details of primers or probes used for qPCR assays included in this study

\begin{tabular}{|c|c|c|c|c|}
\hline Target species & Target gene & Assay type & Primer/Probe sequence & Reference \\
\hline \multirow[t]{2}{*}{ Candidatus Liberibacter asiaticus } & \multirow[t]{2}{*}{ 16S rDNA } & \multirow[t]{2}{*}{ Taqman } & $\begin{array}{l}\text { TCGAGCGCGTATGCGAATAC } \\
\text { GCGTTATCCCGTAGAAAAAGGTAG }\end{array}$ & \multirow[t]{2}{*}[26,27]{} \\
\hline & & & AGACGGGTGAGTAACGCG & \\
\hline \multirow[t]{2}{*}{ Diaphorina citri } & \multirow[t]{2}{*}{ wingless } & \multirow[t]{2}{*}{ Taqman } & $\begin{array}{l}\text { GCTCTCAAAGATCGGTTTGACGG } \\
\text { GCTGCCACGAACGTTACCTTC }\end{array}$ & \multirow[t]{2}{*}[28]{} \\
\hline & & & TTACTGACCATCACTCTGGACGC & \\
\hline Candidatus Carsonella ruddii & 16S rDNA & SYBR & $\begin{array}{l}\text { TGGGAACGCCATATGCTAAT } \\
\text { GTCCCAATGGGTTGTTCATC }\end{array}$ & {$[22]$} \\
\hline Candidatus Profftella armatura & $16 \mathrm{~S}$ rDNA & SYBR & $\begin{array}{l}\text { GCCTTTATGGGTAGGGCTTC } \\
\text { CCGGACTACGATGCACTTTT }\end{array}$ & {$[22]$} \\
\hline Wolbachia & $f t s Z$ & SYBR & $\begin{array}{l}\text { AGCAGCCAGAGAAGCAAGAG } \\
\text { TACGTCGCACACCTTCAAAA }\end{array}$ & {$[22]$} \\
\hline Wolbachia & $w s p$ & $\begin{array}{l}\text { Nested PCR } \\
\text { (outer) } \\
\text { Nested PCR } \\
\text { (inner) }\end{array}$ & $\begin{array}{l}\text { TGGTACAATAAGTGATGAAGAAAC } \\
\text { AAAAATTAAACGCTACTCCA } \\
\text { GGATAGTCCCTTAACAAGAT } \\
\text { TTGATTTCTGGAGTTACATC }\end{array}$ & {$[15]$} \\
\hline
\end{tabular}


sample and standard curve DNA reaction. The qPCR conditions consisted of one cycle of $95^{\circ} \mathrm{C}$ for $10 \mathrm{~min}, 40$ cycles of $95^{\circ} \mathrm{C}$ for $15 \mathrm{~s}$ and $58^{\circ} \mathrm{C}$ (Carsonella and Wolbachia) or $60^{\circ} \mathrm{C}$ (Profftella, CLas and wingless) for $30 \mathrm{~s}$. Dissociation curve analyses were conducted for SYBR green assays. PCR efficiencies were confirmed to be within the range of $90-110 \%$ $\left(R^{2}>99 \%\right)$ for all qPCR assays. The melting curves for all of the SYBR green assays indicated that only the correct amplicons were amplified and no detectable primer dimers were produced.

\section{Data Analysis}

For each sample, the copy numbers of the five target genes in the template DNA were calculated using a previously described method [29]. Subsequently, the endosymbiont copy number was divided by the wingless gene copy number in the same sample. Many of the $D$. citri samples had extremely low CLas infection levels and were therefore difficult to accurately quantify using standard curves. Thus, CLas density across samples was compared by dividing infected $D$. citri into high infection and low infection samples using a density threshold (0.02 copies of CLas 16S rDNA per copy of wingless). Correlations among the densities of different endosymbionts and the effects of locality, gender, and CLas infection on endosymbiont densities were determined/analyzed using SPSS 23 (IBM, Inc.). Statistical analyses (correlation analysis, chi-square test of homogeneity, analysis of variance) were conducted on the endosymbiont densities (measured as copies of endosymbiont per copy of wingless). Endosymbiont densities among field-collected $D$. citri were transformed for statistical analysis using $\log (1+x)$ (Wolbachia) or $\log$ (Carsonella and Profftella) to satisfy the condition of normality for analysis of variance (ANOVA). In some ANOVA tests where the test assumptions were violated after data transformation, a more stringent significant threshold was used $(P<0.01)$; significant factors in these tests were only reported if they meet this threshold. In ANOVA tests where test assumptions were satisfied or only mildly violated, the significant factors were reported when $P<0.05$.

\section{Results}

\section{Presence and Densities of Different Endosymbionts in Field $D$. citri Samples}

Carsonella and Profftella were detected among all 123 D. citri tested. Only three individuals tested negative for the presence of Wolbachia: one male and one female from Lake Alfred, and one male from Clermont. Additional testing of these three samples using a previously described nested conventional PCR assay [15] also failed to detect the endosymbiont (Fig. S1). Overall, Carsonella, Profftella, and Wolbachia were
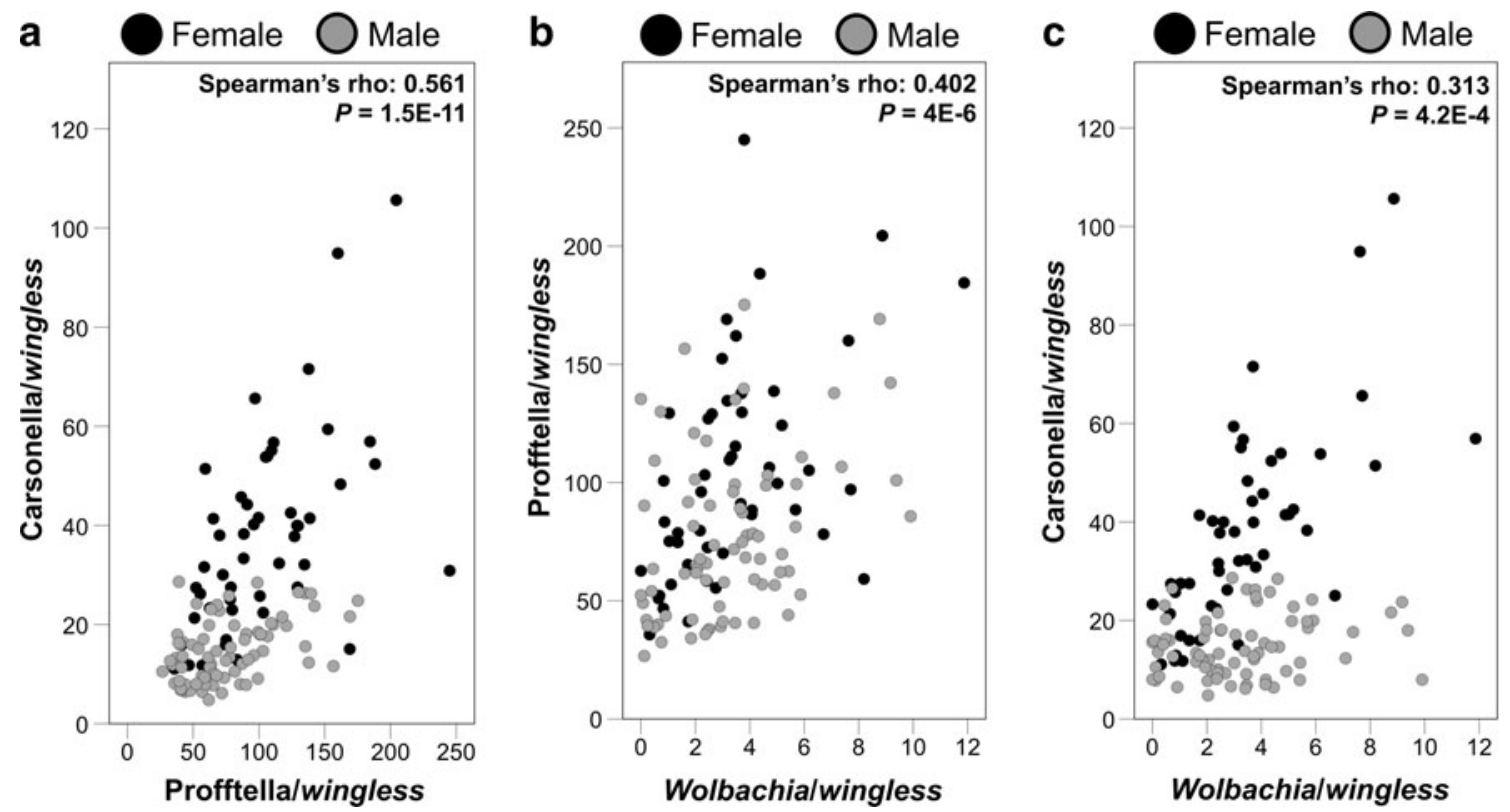

Fig. 1 Scatter plots illustrating correlations among densities of D. citri endosymbionts. a Carsonella and Profftella, b Profftella and Wolbachia, c Carsonella and Wolbachia. Observations are colored (black or gray) by D. citri gender. Correlations of the endosymbiont densities in each plot were determined using pooled data from both genders. Correlation coefficients of endosymbiont densities in female D. citri were 0.594
$(P=7.0 \mathrm{E}-6)$ for Carsonella and Profftella, $0.561(P=2.8 \mathrm{E}-5)$ for Profftella and Wolbachia, and $0.75(P=5.5 \mathrm{E}-10)$ for Carsonella and Wolbachia. Correlation coefficients in males were $0.427(P=1.5 \mathrm{E}-4)$ for Carsonella and Profftella, $0.334(P=0.004)$ for Profftella and Wolbachia, and $0.2(P=0.088)$ for Carsonella and Wolbachia 


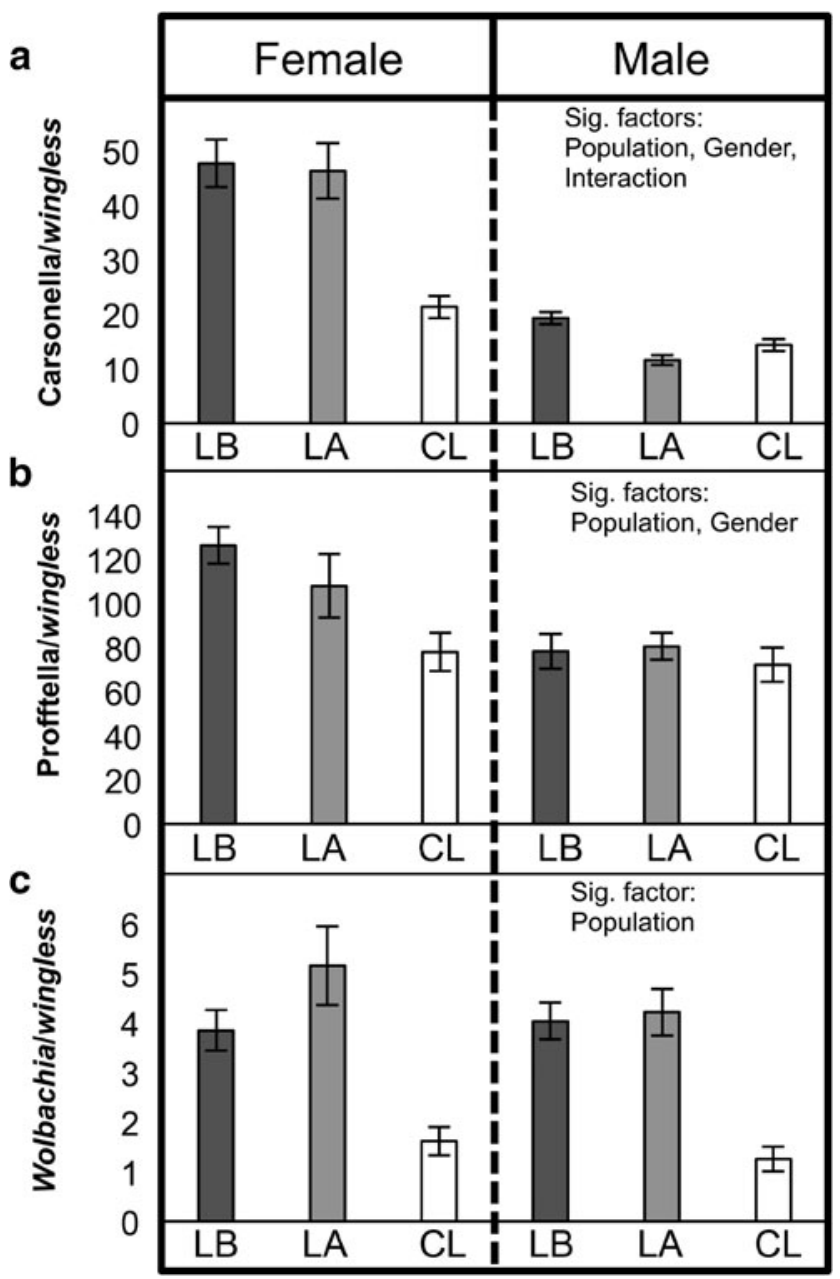

Fig. 2 Densities of a Carsonella, b Profftella, and $\mathbf{c}$ Wolbachia in D. citri adults from different field populations. $L B$ LaBelle; $L A$ Lake Alfred; $C L$ Clermont. Factors significantly associated with each endosymbiont's density are shown on the right. The error bars indicate standard errors. $L B$ LaBelle; $L A$ Lake Alfred; $C L$ Clermont

positively correlated with each other $(P<0.05$; Fig. 1$)$. Positive correlations among the endosymbionts were also detected within each $D$. citri gender, with the exception of Carsonella and Wolbachia in male insects $(P=0.088$; Fig. 1c). The densities of all three endosymbionts were population dependent (Carsonella: $F=22.4, \mathrm{df}=2,117$,

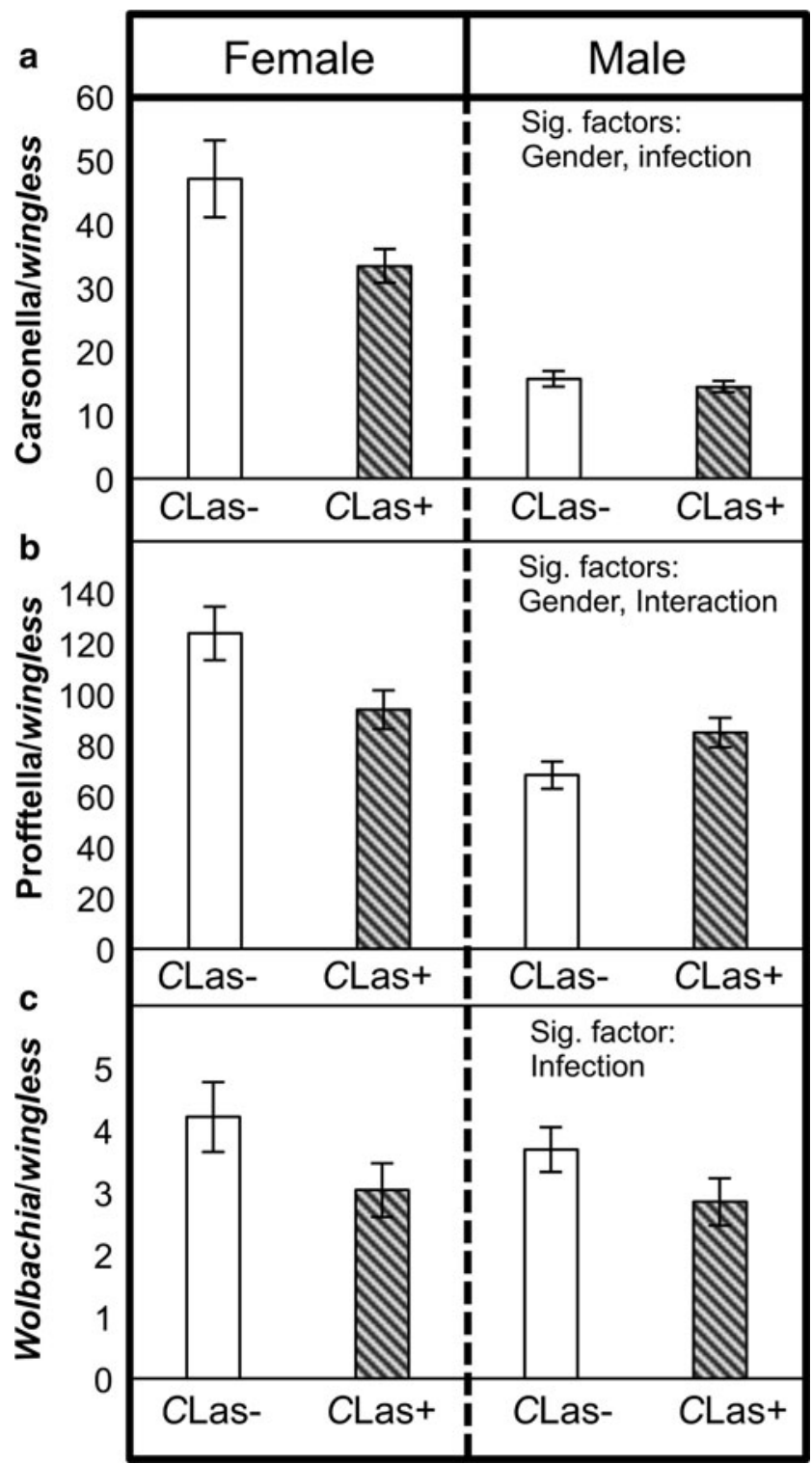

Fig. 4 Densities of a Carsonella, b Profftella, and $\mathbf{c}$ Wolbachia in CLasinfected $\left(C \mathrm{Las}^{+}\right)$or uninfected $\left(C \mathrm{Las}^{-}\right)$field $D$. citri (total $\left.n=123\right)$. Factors significantly associated with each endosymbiont's density are shown on the right. The error bars indicate standard error

$P<0.0001$; Profftella: $F=6.02, \mathrm{df}=2,117, P=0.0032$; Wolbachia: $F=39.49, \mathrm{df}=2,117, P<0.0001)$. The Clermont

Fig. 3 Proportions of D. citri infected with different densities of CLas. ND No CLas detected; LOW CLas densities lower than 0.02 copy of $C$ Las $16 \mathrm{~S}$ rDNA per copy of wingless. HIGH CLas density higher than the threshold. $L B$ LaBelle; $L A$ Lake Alfred; $C L$ Clermont
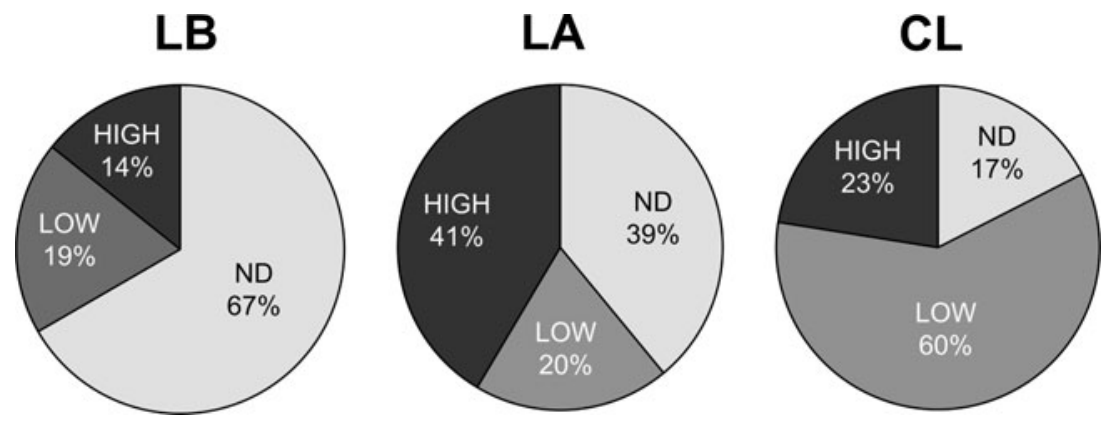
population exhibited the lowest infection densities as compared with the Lake Alfred and LaBelle populations (Fig. 2). $D$. citri gender was significantly associated with the densities of Carsonella and Profftella (Carsonella: $F=163.02, \mathrm{df}=1$, 117, $P<0.0001$; Profftella: $F=15.61, \mathrm{df}=1,117$, $P=0.0001)$. Infection densities were higher in females as compared to male $D$. citri (Fig. 2). Wolbachia densities were not significantly associated with $D$. citri gender $(P>0.05)$.

\section{Infection Prevalence and Density of CLas Among Field D. citri}

Among the 123 field $D$. citri adults sampled, 72 individuals tested positive for the presence of CLas. The three Wolbachianegative individuals (described above) all carried CLas. Cross-population comparisons of the CLas measurements revealed that both the prevalence and the density of $C$ Las infection were population dependent $\left(X^{2}=31.97, \mathrm{df}=4, P<0.05\right.$; Fig. 3). Individuals from the LaBelle population had the lowest CLas infection rate while those from the Clermont population had the highest (Fig. 3). Although the Lake Alfred population had fewer $C$ Las-positive individuals than the Clermont population, there was a higher proportion of $D$. citri infected with high densities of $C$ Las in the Lake Alfred population (high and low infection samples only, Lake Alfred vs Clermont, $X^{2}=9.54, \mathrm{df}=1, P<0.05$ ). When comparing $C$ Las densities across population within each gender, similar patterns were observed (Fig. S2).

\section{Associations Between $C$ Las Infection and Endosymbiont Densities}

To investigate associations between $C$ Las infection and the densities of $D$. citri endosymbionts, $D$. citri samples were categorized according to their $C$ Las infection status (positive or negative) and their endosymbiont densities were compared (Fig. 4). The densities of Carsonella and Wolbachia were significantly higher in CLas-negative compared to CLas-positive D. citri when 123 samples were pooled for two-way ANOVA using gender and infection status as fixed factors (Carsonella: $F=4.96, \mathrm{df}=1,119, P=0.0278 ;$ Wolbachia: $F=7.97, \mathrm{df}=1$,
$119, P=0.0056$ ). There was also a significant interaction effect for gender and infection status on Profftella density (Fig. 4) $(F=10.5, \mathrm{df}=1,119, P=0.0015)$; $C$ Las-infected females had a lower density of Profftella as compared to uninfected females, while the opposite pattern was observed in male D. citri (Fig. 4b). When analyzed in a three-way ANOVA (population $\times$ gender $\times$ infection status), there was a significant population effect across all three endosymbionts (Carsonella: $F=15.96, \mathrm{df}=2,111, P<0.0001$; Profftella: $F=5.1, \mathrm{df}=2,111, P=0.0076$; Wolbachia: $F=26.5, \mathrm{df}=2$, $111, P<0.0001)$, yet the effect of the $C$ Las infection status was no longer significant $(P>0.05)$. When categorizing samples based on the CLas infection density (low, high, or ND) instead of their infection status, the $N D$ (no CLas detected) females tended to have higher endosymbiont densities than the low and high infected females (Table S1). Similar to infection status, the effect of CLas infection density on endosymbiont densities was not significant $(P>0.05)$ when the data were analyzed using a three-way ANOVA including the population factor. Details of the endosymbiont density values can be found in the supplementary material (Table S1).

\section{Endosymbiont Densities in D. citri Populations Reared Under Standardized Conditions}

Laboratory D. citri cultures exhibited Wolbachia densities similar to the patterns observed among field $D$. citri populations. Wolbachia densities were higher in the Lake Alfredderived $D$. citri culture compared to the Clermont $D$. citri culture (one-tailed $t$ test; $t=1.73, \mathrm{df}=20, P<0.05$; Fig. 5). The densities of Carsonella and Profftella did not differ significantly between the $D$. citri cultures from these locations (Fig. 5).

\section{Discussion}

Characterizing factors associated with infection densities of $D$. citri endosymbionts is a necessary step for understanding these bacteria's influence on the psyllid host. Using qPCR and absolute quantitation methods, the present study
Fig. 5 Densities of a Carsonella, b Profftella, and c Wolbachia in laboratory-reared $D$. citri populations. $L A$ Lake Alfred population; $C L$ Clermont population. The asterisk indicates significant difference between populations (one-tailed $t$ test $P<0.05)$. The error bars indicate standard errors
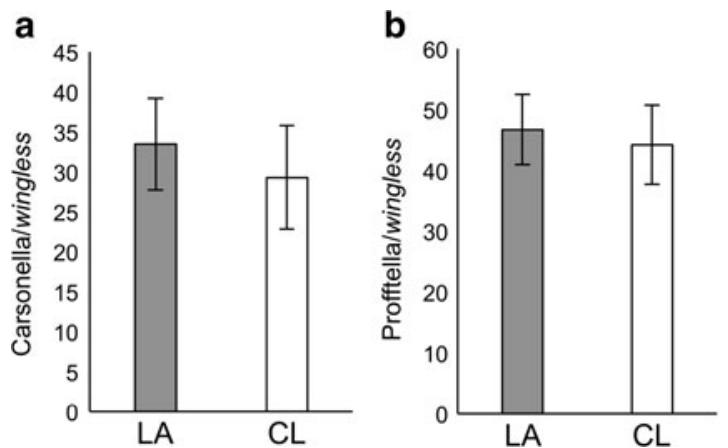
investigated the ecology of $D$. citri endosymbionts across different field populations. The densities of Wolbachia, Profftella, and Carsonella were positively correlated with each other among the field $D$. citri populations, indicating that the growth of an individual endosymbiont is not significantly inhibited by the growth of others. Among the three endosymbionts, there was a stronger correlation (higher coefficient, lower $P$ value) between the densities of Profftella and Carsonella. It is possible that the growth of Profftella and Carsonella, which both inhabit the bacteriomes of $D$. citri, may be more synchronized with each other than with Wolbachia. Unexpectedly, even when assessed using the most sensitive nested-PCR assay, Wolbachia was not detectable in three out of the 123 D. citri analyzed. Previous studies reported $100 \%$ Wolbachia infection among $D$. citri populations in Florida and Brazil $[15,16]$; however, the present work suggests that rare failures of vertical transmission may occur in the field. Examining larger sample sizes or sampling insects during different times of the year may be necessary to detect their occurrence. The finding of $D$. citri individuals infected with $C$ Las, but not Wolbachia, also suggests that Wolbachia infection is not a requirement for $C$ Las acquisition.

The higher density of Profftella and Carsonella in D. citri females may reflect these bacteria's higher rate of abundance increase with age in females relative to males [22]. It is therefore possible that such effects could contribute to the positive correlations and between-gender differences among endosymbiont densities observed in the field samples.

Although the D. citri gender and age could be factors affecting the endosymbionts' densities, it does not completely explain the inter-population variability observed among the field $D$. citri samples. For example, if they were the sole factors determining endosymbiont density, one would expect that the density differences among populations (within gender) would be consistent across all three endosymbionts, since all three endosymbiont densities should gradually increase as their host age [22]. Instead, the data showed that while Carsonella and Profftella densities were similar among the males from all three populations tested, the Wolbachia densities were variable. The CLas infection status of $D$. citri and genotypic variations in $D$. citri and its endosymbionts are possible factors associated with endosymbiont density. Although the possibility that unique endosymbiont- $D$. citri associations (originating from genetic differences) across populations might influence $D$. citri's efficiency to acquire $C$ Las could not be ruled out, the present study falsified the hypotheses that high endosymbiont densities per se either exclude or facilitate CLas acquisition in D. citri (i.e., suggesting that there is no competitive exclusion occurring among endosymbionts and CLas). The results indicated that, although an analysis pooling $D$. citri from different populations suggested that CLas-infected individuals tend to have lower endosymbiont densities compared to uninfected individuals, the same difference was not observed when the population factor was considered (three-way ANOVA model). This finding suggests that populationspecific factors, such as genetic background, may have a stronger effect on the endosymbiont densities.

Interestingly, we found that differences between Wolbachia densities of the Lake Alfred and Clermont $D$. citri were consistent between field samples and those reared under laboratory conditions (standardized diet, age, and environment), which supports the hypothesis of a genetic basis underlying Wolbachia density. In other insect systems, genotypes of the insect and bacteria can determine Wolbachia infection density $[21,30]$. The present study indicates that host-endosymbiont interactions could vary among field $D$. citri populations and may have different repercussions on their biology.

CLas infection prevalence and density varied among the D. citri populations tested. A recent study indicated that D. citri from southern Florida (e.g., LaBelle) had lower CLas infection prevalence compared to northern Florida populations (e.g., Lake Alfred) [31]. Here, the present study not only detected similar differences in the CLas infection prevalence among the three Florida populations, but also showed that the infection intensity also differ among them. A systemic infection by $C$ Las, followed by invasion of the salivary gland could be necessary for $D$. citri to become capable of transmitting the bacteria to healthy plants [14]. A recent study also suggested that $C$ Las density in $D$. citri correlates to their transmission efficiency [32]. Therefore, determining both the density and prevalence of $C$ Las infection in field $D$. citri may allow more accurate monitoring of citrus greening in the field.

Overall, findings from this work indicate that variations between $D$. citri populations appear more important than endosymbiont density in predicting CLas infection. Recognizing the heterogeneous host-microbe interactions in field D. citri and different CLas infection densities across populations could provide venues for investigating D. citri biology and developing strategies for citrus greening management, such as methods to intervene in the pathogen transmission process.

Acknowledgments The authors would like to thank I. P. Foote and A. Rivera for experimental assistance, W. Meyer, B. McLean II, B. McLean III and P. A. Stansly for assistance with insect collections, and A. P. Arp for valuable comments on the study. This project was supported by a United States Department of Agriculture, National Institute for Food and Agriculture (USDA-NIFA) Specialty Crop Research Initiative grant (2012-51181-20086). 
Open Access This article is distributed under the terms of the Creative Commons Attribution 4.0 International License (http:// creativecommons.org/licenses/by/4.0/), which permits unrestricted use, distribution, and reproduction in any medium, provided you give appropriate credit to the original author(s) and the source, provide a link to the Creative Commons license, and indicate if changes were made.

\section{References}

1. Baumann P (2005) Biology of bacteriocyte-associated endosymbionts of plant sap-sucking insects. Annu Rev Microbiol 59:155-189. doi:10.1146/annurev.micro.59.030804.121041

2. Engelstädter J, Hurst GDD (2009) The ecology and evolution of microbes that manipulate host reproduction. Annu Rev Ecol Evol Syst 40:127-149

3. Feldhaar H (2011) Bacterial symbionts as mediators of ecologically important traits of insect hosts. Ecol Entomol 36:533-543. doi:10. 1111/j.1365-2311.2011.01318.x

4. Moran NA, Telang A (1998) Bacteriocyte-associated symbionts of insects. Bioscience 48:295-304

5. Oliver KM, Russell JA, Moran NA, Hunter MS (2003) Facultative bacterial symbionts in aphids confer resistance to parasitic wasps. Proc Natl Acad Sci U S A 100:1803-1807. doi:10.1073/pnas. 0335320100

6. Brownlie JC, Johnson KN (2009) Symbiont-mediated protection in insect hosts. Trends Microbiol 17:348-354. doi:10.1016/j.tim. 2009.05.005

7. Walker T, Johnson PH, Moreira LA, Iturbe-Ormaetxe I, Frentiu FD, McMeniman CJ, Leong YS, Dong Y, Axford J, Kriesner P, Lloyd AL, Ritchie SA, O'Neill SL, Hoffmann AA (2011) The wMel Wolbachia strain blocks dengue and invades caged Aedes aegypti populations. Nature 476:450-453. doi:10.1038/nature10355

8. Blagrove MSC, Arias-Goeta C, Failloux AB, Sinkins SP (2012) Wolbachia strain wMel induces cytoplasmic incompatibility and blocks dengue transmission in Aedes albopictus. Proc Natl Acad Sci U S A 109:255-260. doi:10.1073/pnas. 1112021108

9. Jagoueix S, Bove JM, Garnier M (1994) The phloem-limited bacterium of greening disease of citrus is a member of the alphasubdivision of the Proteobacteria. Int J Syst Bacteriol 44:379-386

10. Halbert SE, Manjunath KL (2004) Asian citrus psyllids (Sternorrhyncha : Psyllidae) and greening disease of citrus: a literature review and assessment of risk in Florida. Fla Entomol 87:330-353. doi:10.1653/0015-4040(2004)087[0330:Acpspa]2.0.Co;2

11. Bové JM (2006) Huanglongbing: a destructive, newly-emerging, century-old disease of citrus. J Plant Pathol 88:7-37

12. Batool A, Iftikhar Y, Mughal SM, Khan MM, Jaskani MJ, Abbas M, Khan IA (2007) Citrus greening disease - a major cause of citrus decline in the world-a review. Hortic Sci 34:159-166

13. Hogenhout SA, el Ammar D, Whitfield AE, Redinbaugh MG (2008) Insect vector interactions with persistently transmitted viruses. Annu Rev Phytopathol 46:327-359. doi:10.1146/annurev. phyto.022508.092135

14. Hall DG, Richardson ML, El-Desouky A, Halbert SE (2013) Asian citrus psyllid, Diaphorina citri, vector of citrus huanglongbing disease. Entomol Exp Appl 146:207-223

15. Guidolin AS, Consoli FL (2013) Molecular characterization of Wolbachia strains associated with the invasive Asian citrus psyllid Diaphorina citri in Brazil. Microb Ecol 65:475-486. doi:10.1007/ s00248-012-0150-7

16. Hoffmann M, Coy MR, Gibbard HNK, Pelz-Stelinski KS (2014) Wolbachia infection density in populations of the Asian citrus psyllid (Hemiptera: Liviidae). Environ Entomol 43:1215-1222. doi:10.1603/En14193

17. Thao ML, Moran NA, Abbot P, Brennan EB, Burckhardt DH, Baumann P (2000) Cospeciation of psyllids and their primary prokaryotic endosymbionts. Appl Environ Microbiol 66:2898-2905. doi:10.1128/Aem.66.7.2898-2905.2000

18. Nakabachi A, Yamashita A, Toh H, Ishikawa H, Dunbar HE, Moran NA, Hattori M (2006) The 160-kilobase genome of the bacterial endosymbiont Carsonella. Science 314:267. doi:10.1126/ science. 1134196

19. Nakabachi A, Ueoka R, Oshima K, Teta R, Mangoni A, Gurgui M, Oldham NJ, van Echten-Deckert G, Okamura K, Yamamoto K, Inoue H, Ohkuma M, Hongoh Y, Miyagishima S, Hattori M, Piel J, Fukatsu T (2013) Defensive bacteriome symbiont with a drastically reduced genome. Curr Biol 23:1478-1484. doi:10.1016/j.cub. 2013.06.027

20. Fagen JR, Giongo A, Brown CT, Davis-Richardson AG, Gano KA, Triplett EW (2012) Characterization of the relative abundance of the citrus pathogen $\mathrm{Ca}$. Liberibacter asiaticus in the microbiome of its insect vector, Diaphorina citri, using high throughput 16S rRNA sequencing. Open Microbiol J 6:29-33. doi:10.2174/ 1874285801206010029

21. Kondo N, Shimada M, Fukatsu T (2005) Infection density of Wolbachia endosymbiont affected by co-infection and host genotype. Biol Lett 1:488-491. doi:10.1098/rsbl.2005.0340

22. Dossi FCA, da Silva EP, Consoli FL (2014) Population dynamics and growth rates of endosymbionts during Diaphorina citri (Hemiptera, Liviidae) ontogeny. Microb Ecol 68:881-889. doi:10. 1007/s00248-014-0463-9

23. Tabachnick WJ (2015) Diaphorina citri (Hemiptera: Liviidae) vector competence for the citrus greening pathogen 'Candidatus Liberibacter asiaticus'. J Econ Entomol 108:839-848. doi:10. 1093/jee/tov038

24. Kolora LD, Powell CM, Hunter W, Bextine B, Lauzon CR (2015) Internal extracellular bacteria of Diaphorina citri Kuwayama (Hemiptera: Psyllidae), the Asian citrus psyllid. Curr Microbiol 70:710-715. doi:10.1007/s00284-015-0774-1

25. Damsteegt VD, Postnikova EN, Stone AL, Kuhlmann M, Wilson C (2010) Murraya paniculata and related species as potential hosts and inoculum reservoirs of 'Candidatus Liberibacter asiaticus', causal agent of huanglongbing. Plant Dis 94:528-533

26. Coy MR, Hoffmann M, Kingdom Gibbard HN, Kuhns EH, Pelz-Stelinski KS, Stelinski LL (2014) Nested-quantitative PCR approach with improved sensitivity for the detection of low titer levels of Candidatus Liberibacter asiaticus in the Asian citrus psyllid, Diaphorina citri Kuwayama. J Microbiol Methods 102:15-22. doi:10.1016/j.mimet.2014.04. 007

27. Li W, Hartung JS, Levy L (2006) Quantitative real-time PCR for detection and identification of Candidatus Liberibacter species associated with citrus huanglongbing. J Microbiol Methods 66:104 115. doi:10.1016/j.mimet.2005.10.018

28. Li W, Duan Y, Brlansky RH, Twieg E, Levy L (2008) Incidence and population of 'Candidatus Liberibacter asiaticus' in Asian citrus psyllids (Diaphorina citri) on citrus plants affected by huanglongbing in Florida. Proceedings of the 2008 International Research Conference Huanglongbing, Orlando, FL, USA

29. Whelan JA, Russell NB, Whelan MA (2003) A method for the absolute quantification of cDNA using real-time PCR. J Immunol Methods 278:261-269. doi:10.1016/S00221759(03)00223-0

30. Mouton L, Henri H, Bouletreau M, Vavre F (2003) Strain-specific regulation of intracellular Wolbachia density in multiply infected 
insects. Mol Ecol 12:3459-3465. doi:10.1046/j.1365-294X.2003. 02015.x

31. Coy MR, Stelinski LL (2015) Great variability in the infection rate of 'Candidatus Liberibacter asiaticus' in field populations of Diaphorina citri (Hemiptera: Liviidae) in Florida. Fla Entomol 98:356-357. doi:10.1653/024.098.0157
32. Ukuda-Hosokawa R, Sadoyama Y, Kishaba M, Kuriwada T, Anbutsu H, Fukatsu T (2015) Infection density dynamics of the citrus greening bacterium "Candidatus Liberibacter asiaticus" in field populations of the psyllid Diaphorina citri and its relevance to the efficiency of pathogen transmission to citrus plants. Appl Environ Microbiol 81:3728-3736. doi:10.1128/AEM.00707-15 